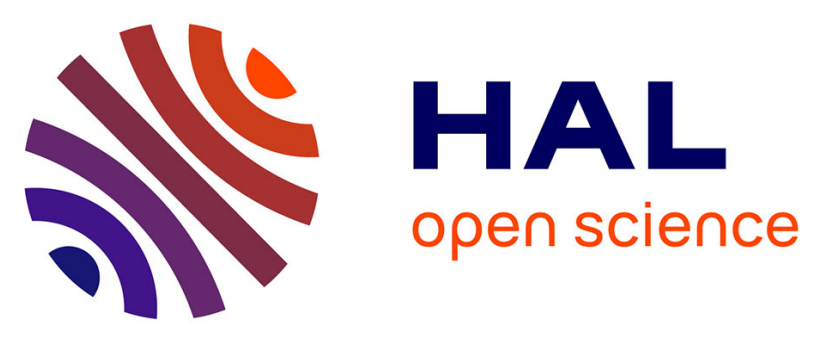

\title{
Influence de l'humidité relative sur l'agressivité et la viabilité des souches de Beauveria bassiana (Bals.) Vuillemin et de Metarhizium anisopliae (Metsch.) Sorokin, hyphomycètes pathogènes de la pyrale du maïs, Ostrinia nubilalis Hübn
}

Guy Riba, Sylvie Marcandier, Jacqueline Goussard

\section{To cite this version:}

Guy Riba, Sylvie Marcandier, Jacqueline Goussard. Influence de l'humidité relative sur l'agressivité et la viabilité des souches de Beauveria bassiana (Bals.) Vuillemin et de Metarhizium anisopliae (Metsch.) Sorokin, hyphomycètes pathogènes de la pyrale du maïs, Ostrinia nubilalis Hübn. Agronomie, 1984, 4 (2), pp.189-194. hal-00884626

\section{HAL Id: hal-00884626 https://hal.science/hal-00884626}

Submitted on 1 Jan 1984

HAL is a multi-disciplinary open access archive for the deposit and dissemination of scientific research documents, whether they are published or not. The documents may come from teaching and research institutions in France or abroad, or from public or private research centers.
L'archive ouverte pluridisciplinaire HAL, est destinée au dépôt et à la diffusion de documents scientifiques de niveau recherche, publiés ou non, émanant des établissements d'enseignement et de recherche français ou étrangers, des laboratoires publics ou privés. 


\title{
Influence de l'humidité relative sur l'agressivité et la viabilité des souches de Beauveria bassiana (Bals.) Vuillemin et de Metarhizium anisopliae (Metsch.) Soro- kin, hyphomycètes pathogènes de la pyrale du maïs, Ostrinia nubilalis Hübn.
}

Guy RIBA \& Sylvic MARCANDIER

avec la collaboration technique de Jacqueline GOUSSARD

I.N.R.A., Station de Recherches de Lutte biologique, La Minière, F 78280 Guyancourt

RÉSUMÉ

\begin{abstract}
Quelques heures d'exposition à des taux d'humidité relative compris entre 10 et 90 p. 100 réduisent considérablement l'activité des hyphomycètes entomopathogènes. Ainsi, la germination des conidies de Beauveria bassiana n'excède pas 20 p. 100 si elles ont été placées 25 j à 30 p. 100 d'hygrométrie. De plus, la durćc de la pathogenèse de $B$. bassiana à l'égard des larves de pyrale du maïs est accrue de $3 \mathrm{j}$ environ lorsque l'humidité relative décroît de 100 à 30 p. 100 ; dans ces conditions le champignon ne peut plus sporuler à la surface des cadavres. Si Metarhizium anisopliae est placé à moins de 90 p. 100 d'hygrométrie, il perd tout pouvoir infecticux à l'égard des œufs de la pyralc du maïs. Par ailleurs, une exposition de $1 \mathrm{i}$ à des taux d'humidité inférieurs à 90 p. 100 suffit à réduire de 85 p. 100 le nombre d'œufs attaqués par ce champignon. Les conséquences de ces phénomènes sur l'épidémiologic des hyphomycètes entomopathogènes sont discutées.
\end{abstract}

Mots clés additionnels : Attaques des ooplaques, germination, sporulation.

Influence of relative humidity on the aggressiveness and viability of conidia of Beauveria bassiana (Bals.) Vuill. and Metarhizium anisopliac (Metsch.) Sorokin, pathogens of Ostrinia nubilalis (Hübn.).

Metarhizium anisopliae could not attack the eggs of Ostrinia nubilalis if the relative humidity of atmosphere was under $90 \%$, even for a few hours. At low relative humidity, Beauveria bassiana was able to penetrate $O$. nubilalis larvae but the LT 50 was much increased and the fungus did not sporulate on the surface of host cadavers. In addition, the viability of conidia of $B$. bassiana and $M$. anisopliae was reduced by relative humidity between 5 and $70 \%$. After 25 days at $30 \%$ RH not more than $20 \%$ of conidia, without any preparation, were able to germinate. In the same way $M$. anisopliae exposed for one day to $30 \% \mathrm{RH}$ at $25^{\circ} \mathrm{C}$ could not attack eggs of $O$. nubilalis. The implications of these results for epizootic development are discussed.

Additional key words : Egg penetration, spore germination, sporulation.

\section{INTRODUCTION}

La mise au point d'un procédé de lutte biologique contre un insecte ravageur des cultures implique une connaissance approfondie de l'influence des facteurs physiques du milieu sur les relations hôte-parasite. De telles études paraissent encore plus justifiées lorsqu'on envisage d'exploiter les potentialités d'auxiliaires tcls que les hyphomycètes entomopathogènes, réputés particulièrement sensibles aux variations de température et d'humidité comme les autres champignons.
En dépit d'une eurythermie assez prononcéc chez la plupart des espèces responsables des muscardines, nous avons pu vérifier, par exemple, qu'une souche de Metarhizium anisopliae (Metsch.) Sorokin, très agressive à l'égard de la pyrale du maïs, Ostrinia nubilalis Hübn. (Lépidoptère Pyralidae), ne peut arrêter rapidement le développement des populations de ce ravageur dans nos conditions climatiques, faute d'une valeur suffisante des températures moyennes dans les conditions de l'expérimentation. En ce qui concerne l'hygrométrie, SCHAERFFENBERG signalait en 1966 que le déclenchement des épizooties naturelles dans les 
populations d'insectes est lié à des situations climatiques ou microclimatiques caractérisées par des humidités relatives élevées. Les essais de modélisation portant sur l'incidence des épizooties à Nomuraea rileyi (Farlow) Samson sur les populations du lépidoptère Noctuidae Anticarsia gemmatalis (Hbn.), dans les cultures de soja en Floride, ont confirmć cette observation (KISH \& ALLEN, 1978). En outre, FERRON (1977) a montré que si l'agressivité de Beauveria bassiana (Bals.) Vuillemin à l'égard des images de la bruche du haricot, Acanthoscelides obtectus Say (Coléoptèrc Curculionidae), n'est pas affectée par des valeurs extrêmes de l'humidité relative, l'automultiplication de l'inoculum entomopathogène par sporulation du mycélium à l'extérieur des cadavres est, au contraire, dépendante d'une hygrométric proche de la saturation.

Etant donné la grande sensibilité des différents stades dc la pyrale du maïs aux muscardines provoquécs par les hyphomycètes entomopathogènes (RIBA et al., 1983), l'analyse des perspectives d'utilisation pratique d'un tel procédé de lutte microbiologique contre ce ravageur nous a conduits à étudier l'influence de l'humidité relative sur l'agressivité, la viabilité et la sporulation des 2 espèces les plus agressives, $B$. bassiana et $M$. anisopliae.

\section{MATÉRIEL ET MÉTHODES}

Les souches de champignons entomopathogènes étudićcs ont été isolées, pendant l'annéc 1980, des populations de pyrale du maïs présentes en Beauce. Elles sont enregistrées sous les références $B$. bassiana 147 et $M$. anisopliae 139 dans la mycothèque du laboratoirc. Il a été vérifić que les chenilles de pyrale sont particulièrement sensibles à l'isolat B. bassiana 147 alors qu'au contraire ce sont les œufs qui sont préférentiellement attaqués par l'isolat $M$. anisopliae 139 (RIBA et al., 1983). C'est pourquoi, pour chaque espèce de champignon, les expériences, n'ont concerné que le stade le plus sensible.

Les différentes valeurs étudiées de l'humidité relative sont obtenues en enceinte close en présence d'eau pure ou de cristaux anhydres de $\mathrm{CaCl}_{2}$ pour les extrêmes $(0$ et $100 \mathrm{p}$. $100 \mathrm{HR}$ ), de solutions saturées de $\mathrm{Na}_{2} \mathrm{CO}_{3}, 10 \mathrm{H}_{2} \mathrm{O}$ et de $\mathrm{CaCl}_{2} 6 \mathrm{H}_{2} \mathrm{O}$ pour les valeurs voisines respectivement de 90 et de 30 p. 100 HR ou d'eau glycérolée pour obtenir 95 p. 100 HR (O'BRIEN, 1948). Les faibles variations du degré hygrométrique en fonction de ces températures sont considérées comme négligeables, compte tenu de la gamme des hygrométries étudiées.

Les techniques employées ont permis d'évaluer, d'une part, l'influence de l'hygrométrie sur l'agressivité de $B$. bassiana à l'égard des larves ou de $M$. anisopliae à l'ćgard des œufs, d'autre part, de mesurer l'impact de ce facteur sur la viabilité des conjdies.

\section{A. Mesure de l'agressivité de $B$. bassiana et de M. anisopliae}

L'agressivité des propagules de $B$. bassiana à l'égard des chenilles de pyrale est évaluće en contaminant par essai biologique des larves diapausantes au $5^{c}$ stade larvaire, réparties en 4 lots de 20 individus ct soumises à la pulvérisation, sous conditions contrôlées et reproductibles, d'unc suspension aqueuse de conidies à $10^{7}$ spores $/ \mathrm{ml}$, de façon à obtenir un dépôt uniforme de $2.10^{4}$ spores $/ \mathrm{cm}^{2}$ selon la technique proposée par BURGERJON (1956). Lc matériel biologique ainsi contaminé est placé sous diverses conditions de température et d'humidité. Etant donné les actions combinées de l'hygrométric, de la température et du temps, les valcurs respectives de ces paramètres seront précisées pour chaque expérience.

L'agressivité de $M$. anisopliae à l'égard des œufs de pyralc est mesurée après contamination d'ooplaques âgées au plus de $24 \mathrm{~h}$ : celles-ci, fixées sur des languettes de papier, sont trempées pendant $30 \mathrm{~s}$ dans des suspensions de conidies de titre connu. Pour chaque variante 4 lots de 6 ooplaques sont examinés. Après 5 j d'incubation dans des conditions hygrométriques déterminécs et variables suivant le protocole expérimental adopté, les œufs éclos ou atteints de mycosc sont contrôlés par observation sous la loupe binoculaire.

\section{B. Mesure de la viabilité de $B$. bassiana et de M. anisopliae}

La viabilité des conidies de $B$. bassiana est déterminéc par calcul du pourcentage de germination des spores placées, pendant des délais variables, en incubation dans des enceintes à humidité relative définic, à la températurc de $25^{\circ} \mathrm{C} \pm 1{ }^{\circ} \mathrm{C}$. Les conidies soumises à cette épreuve sont recueillies par grattage à l'anse de platine à la surface d'un milieu de culture gélosé. Elles se présentent sous la forme d'une poudre humide, à 93 p. 100 de teneur en cau. Cette poudre humide n'a pas ćté desséchée avant d'être répartic dans les différentes enceintes. Le pourcentage de germination est défini par le rapport entre le nombre de conidies dont la longueur du tube germinatif est supérieure ou ćgale à 2 fois le grand diamètre de la spore, d'une part, et le nombre total de propagules observées, d'autre part. Cette observation porte sur 4 fois 100 conidies incubées pendant 24 h à $25^{\circ} \mathrm{C}$ à la surface d'un milieu complet gélosé coulé en boîte de Petri $($ milieu complet $=$ glucose 1 p. 100 ct extrait de levure 0,5 p. 100).

Pour $M$. anisopliae, la viabilité des propagules a été calculéc par la mesure de la rémanence de leur agressivité à l'égard des œufs de pyrale. A cet effet, une suspension de conidies est pulvérisće, dans les mêmes conditions expérimentales que précédemment, à la surface de feuilles de papier qui sont ensuite mises en incubation, pendant des délais variables, à $25^{\circ} \mathrm{C}$ dans des enccintes à hygrométrie réguléc. A des intervalles de temps connus, unc de ces feuilles cst prélevée et placée à la face supérieure d'une cage de ponte pendant $24 \mathrm{~h}$. Après la ponte des papillons, les ooplaques déposées sont individualisées, en découpant avec une paire de ciseaux le papier support, et mises en incubation pendant $5 \mathrm{j}$ à $25^{\circ} \mathrm{C}$ dans une enceinte à hygrométric saturée $(=100$ p. $100 \mathrm{HR})$. Les cufs éclos ou atteints de mycose sont alors dénombrés sous la loupe binoculairc.

\section{RÉSULTATS}

\section{A. Influence de l'humidité relative sur l'agressivité des champignons entomopathogènes}

\section{Cas de la muscardine blanche à $\mathrm{B}$. bassiana}

Après pulvérisation d'une suspension du germe titrant $1.10^{7}$ conidies $/ \mathrm{ml}$ à la surface de chenilles diapausantes, celles-ci sont mises en élevage dans des enccintes régulées respectivement à 100 et 30 p. $100 \mathrm{HR}$ aux températures de $15^{\circ} \mathrm{C}$ et de $25^{\circ} \mathrm{C}$. Des lots non traités subissent les mêmes conditions expérimentales (tabl. 1).

On constate que la contamination artificicle par $B$. bassiana provoque un très net accroissement de la mortalité totale. Cette mortalité est obtenue d'une façon indépen- 
TABLEAU 1

Influence combinée de l'hygrométrie et de la température sur la sensibilité des chenilles diapausantes de pyrale à la muscardine blanche à B. bassiana.

Combined influence of relative humidity and temperature on the susceptibility of diapausing larvae of $\mathrm{O}$. nubilalis to $\mathrm{B}$. bassiana.

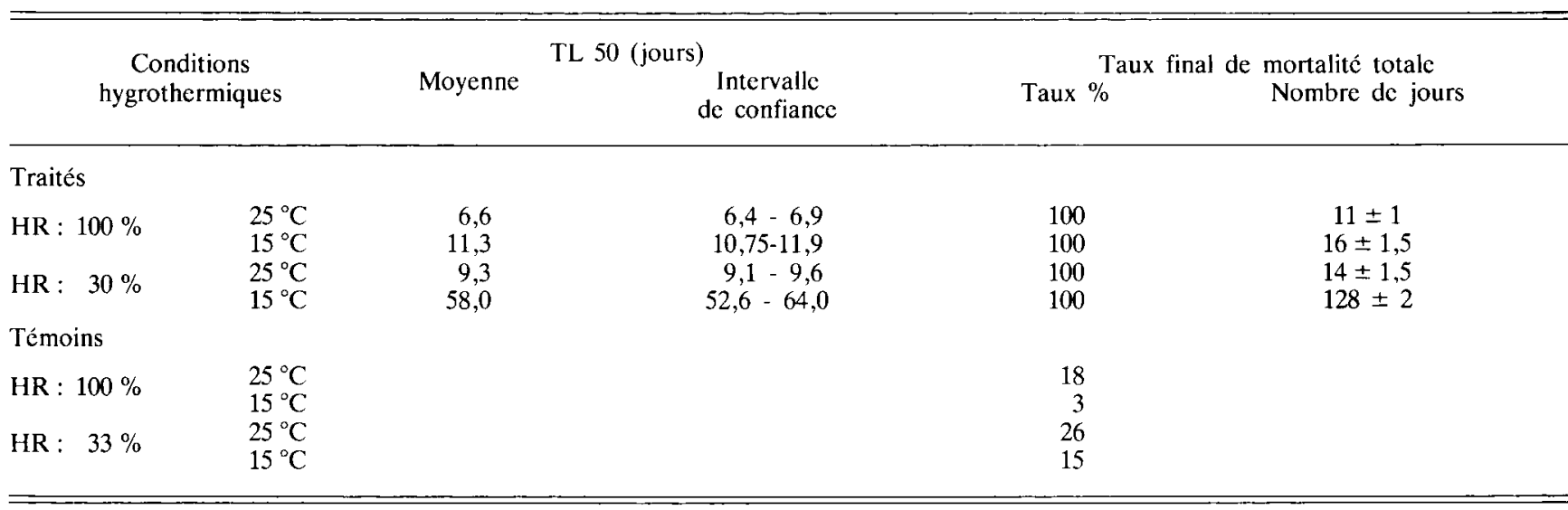

TABLEAU 2

Sensibilité des aeufs de pyrale à Metarhizium anisopliae en fonction des valeurs de l'hygrométrie relative de l'ambiance (contamination assurée par trempage dans une suspension aqueuse de conidies titrant $1.10^{7}$ spores par $\mathrm{ml}$ ).

Susceptibility of eggs of $\mathrm{O}$. nubilalis to $\mathrm{M}$. anisopliac to various relative humidity values (eggs treated by dipping in conidial suspension at $10^{7}$ spores $(\mathrm{ml})$

\begin{tabular}{|c|c|c|c|c|c|c|}
\hline \multirow{2}{*}{$\begin{array}{l}\text { Humidité relative } \\
\text { (pourcentage) }\end{array}$} & \multicolumn{3}{|c|}{ M. anisopliae $139 \%$ d'œufs } & \multicolumn{3}{|c|}{ Témoin \% d'œufs } \\
\hline & éclos & non éclos & mycosés & éclos & non éclos & mycosés \\
\hline 100 & 0 & 100 & 100 & 100 & 0 & 0 \\
\hline 90 & 92 & 8 & 0 & 100 & 0 & 0 \\
\hline 76 & 100 & 0 & 0 & 100 & 0 & 0 \\
\hline 30 & 100 & 0 & 0 & 92 & 8 & 0 \\
\hline 0 & 20 & 80 & 0 & 85 & 15 & 0 \\
\hline
\end{tabular}

dante des taux d'humidité relative. Par contre, les processus pathologiques sont ralentis aux faibles hygrométries, lc temps létal 50 (TL 50) passant respectivement de 6,6 à $9,3 \mathrm{j}$ à $25^{\circ} \mathrm{C}$ pour des humidités relatives de 100 et $30 \mathrm{p} .100 \mathrm{HR}$, alors qu'à $15^{\circ} \mathrm{C}$ le phénomène s'amplific, le temps létal 50 passant de 11,3 à $58,0 \mathrm{j}$ dans les mêmes conditions cxpérimentales.

On a, en outre, confirmé les observations de FERRON (1977) concernant l'influence de l'humidité sur la sporulation du champignon à l'extérieur des cadavres de chenilles transformées en momies par le mycélium. En effet, le développement externe de ce mycélium et la formation des conidies ne sont obtenus que si les cadavres sont placés à une hygrométrie saturante. Dans des conditions d'humidité relative basse, le champignon survit cependant: après 3 mois d'incubation à $30 \mathrm{p} .100 \mathrm{HR}$ et $25^{\circ} \mathrm{C}$, il se développe à l'extérieur des momies et sporule 3 à $6 \mathrm{j}$ après son transfert cn humidité saturéc (100 p. $100 \mathrm{HR})$.

Pour cette raison, seuls les pourcentages de mortalité totale ont été analysés dans cette expérience puisque à 33 p. $100 \mathrm{HR}$ par excmple, aucun cadavre ne se recouvre de champignon.

2. Cas de la muscardine verte à M. anisopliac

Après contamination par trempage dans une suspension titrant $1.10^{7}$ conidies par $\mathrm{ml}$, les ooplaques sont incubées respectivement pendant 3 j à $0,30,76,90$ et 100 p. $100 \mathrm{HR}$, puis sont toutes placées pendant $2 \mathrm{j}$ supplémentaires dans une enceinte à 100 p. $100 \mathrm{HR}$ pour éviter l'influence néfaste de la dessiccation sur l'éclosion des oufs. Les différentes variantes expérimentales sont placées à la même température de $25^{\circ} \mathrm{C} \pm 1{ }^{\circ} \mathrm{C}$. Les résultats obtenus sont indiqués dans le tableau 2. Ils montrent que le développement de la muscardine n'est assuré qu'en présence d'une hygrométric saturante permanente. On remarque que l'éclosion des œufs de pyrale est limitée seulement par la valeur la plus basse de l'hygrométrie $(0 \mathrm{p} .100 \mathrm{HR})$ sans manifestation de la mycose.

Dans une $2^{\mathrm{e}}$ expérience (tabl. 3), le séjour des ooplaques contaminces aux diverses hygrométrics précédemment indiquées a été réduit respectivement à $15 \mathrm{~h}, 1,2$ et $3 \mathrm{j}$ avant leur transfert dans une enceinte à hygrométrie saturante de façon que le délai total d'incubation soit, dans tous les cas, de $6 \mathrm{j}$. On constate alors que, même après seulement $15 \mathrm{~h}$ d'incubation à 90 p. $100 \mathrm{HR}$, le champignon n'infecte que 10 p. 100 des œufs. Le phénomène observé se manifeste donc très rapidement.

\section{B. Influence de l'humidité relative sur la viabilité des champignons entomopathogènes}

\section{Cas des conidies de B. bassiana}

Des conidies non déshydratées sont placées à $25^{\circ} \mathrm{C}$ dans des enceintes à hygrométric régulée respectivement à 0,30 , 76,90 et 100 p. 100 pendant $3,5,8,19$ et 25 j. Conscrvées $25 \mathrm{j}$ à ces hygrométries puis ensemencées sur milicu nutritif 
TABLEAU 3

Influence de la durée d'exposition à des humidités relatives ambiantes différentes sur l'agressivité de M. anisopliae à l'égard des aeufs de pyrale du maïs placés à $25{ }^{\circ} \mathrm{C}$. Les pourcentages de mycose sont évalués ie $\sigma^{e} j$ après le traitement à $10^{7}$ spores $/ \mathrm{ml}$.

Influence of relative humidity on the aggressiveness of $\mathrm{M}$. anisopliac to eggs of $\mathrm{O}$. nubilalis at $25^{\circ} \mathrm{C}$. Percent mycosis calculated 6 days after treatment at $10^{7}$ spores $/ \mathrm{ml}$.

\begin{tabular}{rrrrrrr}
\hline Duréc & HR \% & \multicolumn{5}{c}{ Eufs mycosés (pourcentages) } \\
& & 100 & 90 & 76 & 30 & 0 \\
\hline & & 100 & 10 & 5 & 5 & 0 \\
$15 \mathrm{~h}$ & 100 & 8 & 3 & 0 & 0 \\
$1 \mathrm{j}$ & 100 & 6 & 0 & 0 & 0 \\
$2 \mathrm{j}$ & 100 & 0 & 0 & 0 & 0 \\
$3 \mathrm{j}$ & & & & \\
\hline
\end{tabular}

en boîte de Petri, on remarque que la viabilité des conidies est très sensiblement affectée par les valeurs de l'humidité relative voisines de 30 p. $100 \mathrm{HR}$ (fig. 1) : en effet, dans ces conditions, le pourcentage de germination fluctue entre 18 et $10 \mathrm{p} .100$ après $24 \mathrm{~h}$ de culture, alors qu'il dépasse $65 \mathrm{p}$. 100 pour les valeurs supérieures ou égales à $76 \mathrm{p}$. $100 \mathrm{HR}$ ou au contraire voisines de 0 p. $100 \mathrm{HR}$.

\section{Cas des conidies de M. anisopliae}

L'infection des œufs de la pyrale du maïs par des conidies de $M$. anisopliae est extrêmement dépendante des conditions d'humidité relative. C'est pourquoi nous avons évalué la rémanence des conidies de $M$. anisopliae par la mesure de la persistance de leur pouvoir infectieux sur les oufs de l'hôte.

Pour cela, des feuilles de papier contaminées par une suspension de conidies sont incubées pendant 1, 2, 3, 7, 9 et $15 \mathrm{j}$ à 20,25 et $30^{\circ} \mathrm{C}$ dans des enccintes à hygrométrie régulée à $0,30,90$ et 100 p. $100 \mathrm{HR}$, avant d'être utilisées comme support de ponte par les papillons de pyrale du maiss. Les pourcentages d'œufs éclos ou morts après $5 \mathrm{j}$ d'incubation à $100 \mathrm{p}$. $100 \mathrm{HR}$ et à $25^{\circ} \mathrm{C}$, suivant ces différentes variantes expérimentales, sont présentés dans le tableau 4.

On remarque, d'une façon générale, que la rémanence du germe est d'autant plus grande que la températurc d'incubation est plus basse : après $15 \mathrm{j}$ d'incubation à $100 \mathrm{p} .100 \mathrm{HR}$, les pourcentages d'œufs éclos sont respectivement de 63,26 et $18 \mathrm{p} .100$ pour les températures de 30,25 et $20^{\circ} \mathrm{C}$. D'autre part, cette rémanence est d'autant plus grande que les valeurs de l'hygrométrie sont plus élevées (fig. 2) : après $15 \mathrm{j}$ d'incubation à $20^{\circ} \mathrm{C}$, les pourcentages d'œufs éclos sont respectivement de 94, 100, 6 et 18 p. 100 pour les humidités relatives de $0,30,90$ et 100 p. 100 . Ces différences sont encore plus nettes à $25^{\circ} \mathrm{C}$, valcur pour laquelle on observe 86 p. 100 des aufs éclos à 90 p. $100 \mathrm{HR}$ contre seulement 26 p. 100 à 100 p. $100 \mathrm{HR}$.

Les valeurs intermédiaires de l'humidité relative $(\times 30$ p. $100 \mathrm{HR}$ ) paraissent plus défavorables à la survie du germe que les valeurs extrêmes, même si celles-ci sont proches ou égales de la dessication. Ainsi après $7 \mathrm{j}$ d'incubation du champignon à $20^{\circ} \mathrm{C}$ et $33 \mathrm{p} .100 \mathrm{HR}, 100 \mathrm{p} .100 \mathrm{des}$ œufs pondus sur les feuilles contaminées avec lc germe éclosent alors cue ce pourcentage n'est que de 73 p. 100 à 0 p. $100 \mathrm{HR}$.

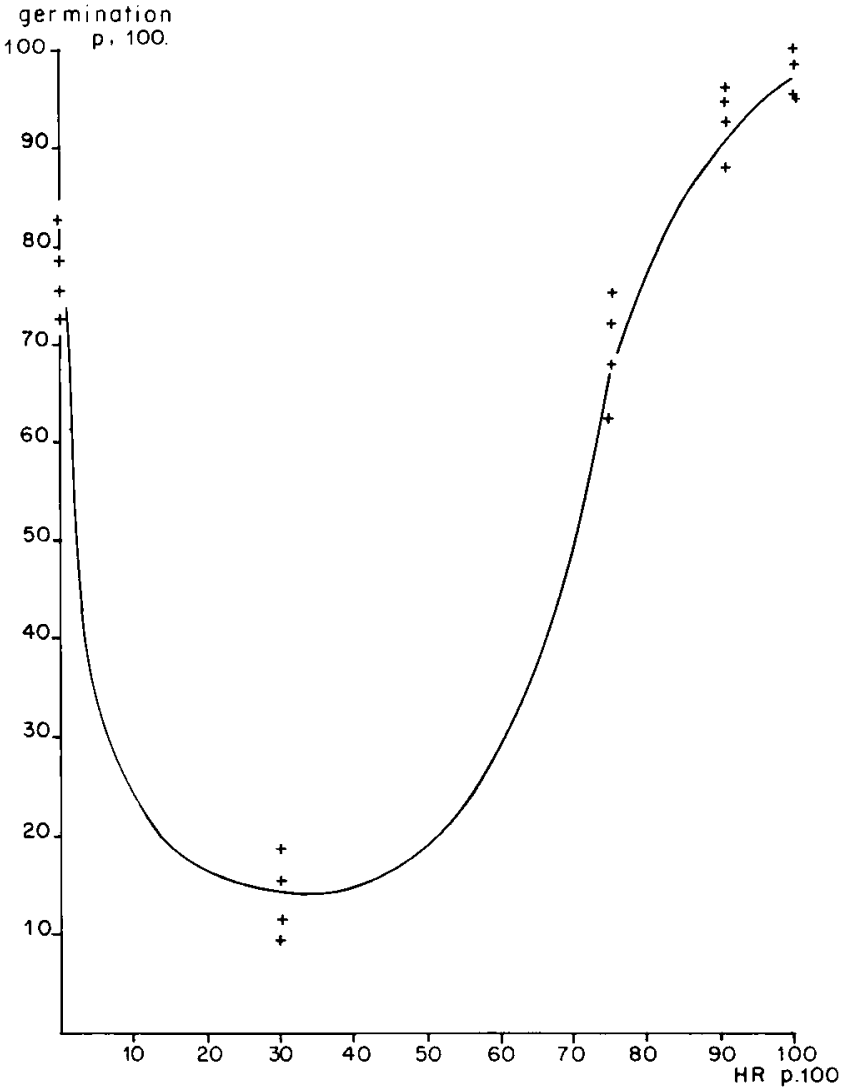

Figure 1

Influence de l'humidité relative sur le pouvoir de germination de conidies de Beauveria bassiana. La mesure est effectuée après 25 j de conservation des spores à $25^{\circ} \mathrm{C}$ aux conditions hygrométriques citées.

Influence of relative humidity on conidial germination of Beauveria bassiana after 25 days'exposure at $25^{\circ} \mathrm{C}$.

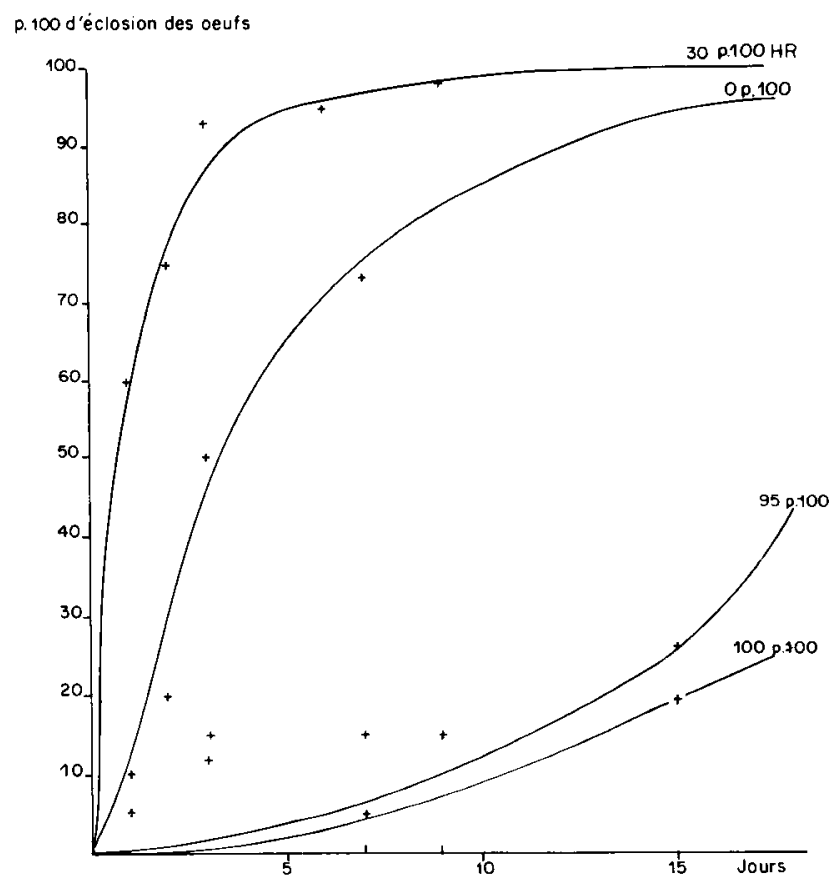

Figure 2

Persistance de l'agressivité des conidies de $\mathrm{M}$. anisopliae en fonction de l'humidité relative ambiante à $20^{\circ} \mathrm{C}$.

Influence of relative humidity on the persistance of the aggressiveness of the conidia of $\mathrm{M}$. anisopliae at $20^{\circ} \mathrm{C}$. 
TABLEAU 4

Influence des conditions hygrothermiques sur la persistance de l'infectivité de M. anisopliae à l'égard des aeufs de pyrale. Influence of relative humidity on the stability of aggressiveness of $\mathrm{M}$. anisopliac to eggs of $\mathrm{O}$. nubilalis.

\begin{tabular}{|c|c|c|c|c|c|c|c|c|c|c|c|c|c|c|c|c|c|c|c|c|c|c|c|c|}
\hline \multirow[b]{3}{*}{ Durée (jours) } & \multicolumn{8}{|c|}{$20^{\circ} \mathrm{C}$} & \multicolumn{8}{|c|}{$25^{\circ} \mathrm{C}$} & \multicolumn{8}{|c|}{$30{ }^{\circ} \mathrm{C}$} \\
\hline & \multicolumn{2}{|c|}{100} & \multicolumn{2}{|c|}{90} & \multicolumn{2}{|c|}{30} & \multicolumn{2}{|c|}{0} & \multicolumn{2}{|c|}{100} & \multicolumn{2}{|c|}{90} & \multicolumn{2}{|c|}{30} & \multicolumn{2}{|c|}{0} & \multicolumn{2}{|c|}{100} & \multicolumn{2}{|c|}{90} & \multicolumn{2}{|c|}{30} & \multicolumn{2}{|c|}{0} \\
\hline & $\mathrm{E}$ & $\mathbf{M}$ & $\mathrm{E}$ & $\mathbf{M}$ & $\mathrm{E}$ & $\mathbf{M}$ & $\mathrm{E}$ & $\mathbf{M}$ & $\mathrm{E}$ & $\mathrm{M}$ & $\mathrm{E}$ & $\mathbf{M}$ & $\mathrm{E}$ & $M$ & $\mathrm{E}$ & $\mathbf{M}$ & $\mathrm{E}$ & $\mathbf{M}$ & $\mathrm{E}$ & $\mathbf{M}$ & $\mathrm{E}$ & $\mathbf{M}$ & $\mathrm{E}$ & $\mathbf{M}$ \\
\hline 1 & 10 & 90 & 5 & 95 & 90 & 10 & 25 & 75 & 0 & 100 & 25 & 75 & 95 & 5 & 67 & 33 & 0 & 100 & 12 & 88 & 95 & 5 & 65 & 35 \\
\hline 2 & 10 & 90 & 5 & 95 & 95 & 5 & 49 & 51 & 0 & 100 & 30 & 70 & 100 & 0 & 93 & 7 & 5 & 95 & 26 & 74 & 97 & 3 & 76 & 24 \\
\hline 3 & 12 & 88 & 5 & 95 & 100 & 0 & 57 & 43 & 0 & 100 & 37 & 63 & 100 & 0 & 95 & 5 & 25 & 7 & 55 & 45 & 100 & 0 & 100 & 0 \\
\hline 7 & 15 & 85 & 55 & 45 & 100 & 0 & 73 & 27 & 5 & 95 & 42 & 58 & 100 & 0 & 97 & 3 & 40 & 60 & 75 & 25 & 100 & 0 & 100 & 0 \\
\hline 9 & 15 & 85 & 63 & 37 & 100 & 0 & 80 & 20 & 11 & 89 & 58 & 42 & 100 & 0 & 9 & 1 & 45 & 55 & 87 & 13 & 100 & 0 & 100 & 0 \\
\hline 15 & 18 & 82 & 67 & 33 & 100 & 0 & 94 & 6 & 26 & 74 & 86 & 14 & 100 & 0 & 100 & 0 & 63 & 37 & 97 & 3 & 100 & 0 & 100 & 0 \\
\hline
\end{tabular}

$\mathrm{E}=$ pourcentage éclosion - percent hatching.

$\mathrm{M}=$ pourcentage d'œufs mycosés - percent of mycosed eggs.

Complémentairement aux résultats du tableau 2, la figure 2 met en évidence la forte influence de l'humidité relative sur le maintien de l'infectivité de $M$. anisopliae à l'égard des œufs de la pyrale du maïs. L'effet néfaste des humidités relatives ambiantes intermédiaires sur la persistance de l'infectivité est donc très rapide.

\section{DISCUSSION ET CONCLUSION}

Les résultats présentés ci-dessus montrent que l'hygrométrie influence l'activité du germe puisque la germination, le pouvoir infectieux et la viabilité des conidies sont dépendants des taux d'humidité relative. Les conséquences de ces phénomènes sur l'épidémiologie de ces germes et la stratégie de leur utilisation en lutte biologique seront discutées.

Les faibles hygrométries ralentissent la germination des spores de B. bassiana (WALSTAD et al., 1970). Nos résultats complètent cette affirmation puisque la pathogenèse est ralentie lorsque l'humidité relative décroît. Ainsi la différence des temps létaux 50 p. 100 entre 100 et 30 p. $100 \mathrm{HR}$ est de $46,7 \mathrm{j}$ à $15^{\circ} \mathrm{C}$. Ferron (1977) et Kramer (1980) n'ont pas observé ce phénomène ; mais comme ces auteurs, nous confirmons que le pourcentage de mortalité de l'hôte n'est pas affecté par les variations de l'humidité ambiante.

Quelques heures d'exposition à des ambiances où le degré hygrométrique est inférieur à $90 \mathrm{p} .100$ suffisent pour empêcher $M$. anisopliae d'attaquer les œufs de pyrale. On peut penser que cette surprenante sensibilité est duc à l'absence de «niche micro-climatique» à la surface de l'ooplaque. A l'opposé, les replis tégumentaires créent des micro-environnements sursaturés et permettent ainsi au champignon de pénétrer la chenille. WAGGONER (1965) soulignait aussi l'importance du microclimat à la surface de la feuille du végétal, dans les infections cryptogamiques. Outre l'agressivité, la viabilité des conidies est fortement influencée par le taux d'humidité relative. Ainsi, après $25 \mathrm{j}$ de conservation à $20^{\circ} \mathrm{C}$ et $30 \mathrm{p} .100$ d'hygrométrie, seuls 15 p. 100 des conidies de $B$. bassiana peuvent germer. La conservation des conidies, en l'absence de toute formulation, doit se faire en atmosphère saturante ou en atmosphère très sèche dont le taux d'hygrométrie est inférieur à 5 p. 100.

Des conidies de $M$. anisopliae placées 48 h à $25^{\circ} \mathrm{C}$ et $30 \mathrm{p}$. 100 d'hygrométrie ne sont pas tuées et pourtant cela suffit pour qu'elles ne puissent plus attaquer les œufs de la pyrale du maïs, même si elles sont mises en atmosphère saturante immédiatement après ce délai.

Le développement des processus ćpizootiologiques est fortement régulé par les conditions hygrométriques (IGNOFFO, 1981 ; KISH \& ALLEN, 1978). Le champignon ne sporule à la surface des cadavres mycosés que lorsque l'humidité est saturante. Notons cependant que le germe survit au moins 3 mois dans l'insecte, même dans des conditions sèches. De plus, la mort des larves attaquées par le germe est différée de quelques jours en atmosphère non saturante tandis que l'infection des cufs de pyrale par $M$. anisopliae est devenue impossible. A la vue de ces résultats expérimentaux, on ne peut donc pas envisager de réduire dans la nature des populations de pyrale du maïs en appliquant ces champignons sur les ooplaques. Dans ces conditions, seuls les premicrs stades larvaires pourront ĉtre tués par le champignon.

Reçu le 9 mai 1983. Accepté le 20 septembre 1983.

\section{RÉFÉRENCES BIBLIOGRAPHIQUES}

Burgerjon A., 1956. Pulvérisation ct poudrage au laboratoire des préparations pathogènes insecticides. Ann. Epiphyt., 4, 677-686. Ferron P., 1977. Influcnce of relative humidity on the development of fungal infection caused by Beauveria bassiana (Fungi Imperfecti, Moniliales) in imagines of Acanthoscelides obtectus (Col. : Bruchidae). Entomophaga, 22, 393-396.
Ignoffo C. M., 1981. The fungus Nomuraea rileyi as a microbial insecticide, p. 513-538. In Ed. H.D. Burges. Microbial controled of pest and plant diseases 1970-1980. Academic Press.

Kish L. P., Allen G. E., 1978. The biology and ccology of Nomuraea rileyi and a program for predicting its incidence on Anticarsia gemmatilis in soybean. I.F.A.S. Univ. of Florida, 48 p. 
Kramer J. P., 1980. The house fly mycosis caused by Entomophthora muscae: influence of relative humidity on infectivity and conidial germination. J.N.Y. Entomol. Soc., 88, 236-240.

O'Brien F., 1948. The control of humidity by saturated salt solutions. J. Sci. Instrum., 25, 73-76.

Riba G., Marcandier S., Richard G., Larget I., 1983. Sensibilité de la pyrale du maïs (Ostrinia nubilalis Hübn. Lep. Pyralidae) aux hyphomycètes entomopathogènes. Entomophaga, 28 (1), 55-64.
Schaerffenberg B., 1966. Können Pilzepidemien bei Insekten künstlich hervorgerufen werden. Z. Angew. Entomol., 58, 362-372. Waggoner P. E., 1965. Microclimate and plant disease. Annu. Rev. Phytopathol., 3, 103-126.

Walstad J. D., Anderson R. F., Stambaugh W. I., 1970. Effects of environmental conditions on two species of muscardine fungi (Beauveria bassiana and Metarhizium anisopliae). J. Invert. Pathol., 16, 221-226. 